\title{
COMPARISON OF TRADITIONAL AND MACHINE LEARNING BASE METHODS FOR GROUND POINT CLOUD LABELING
}

\author{
S. M. Ayazi *, M. SaadatSeresht \\ School of Surveying and Geospatial Engineering, College of Engineering, University of Tehran, Iran - (smayazi, \\ msaadat)@gmail.com
}

Commission III, WG III/2

KEY WORDS: Point Cloud, Filtering, Machine Learning, DTM Extraction,

\begin{abstract}
:
Today, a variety of methods have been proposed by researchers to distinguish ground and non-ground points in point cloud data. Most fully automated methods have a common disadvantage which is the lack of proper algorithm response for all areas and levels of the ground, so most of these algorithms have good outcomes in simple landscapes but encounter problems in complex landscapes. Point cloud filtering techniques can be divided into two general rule-based and novel methods. Today, the use of machine learning techniques has improved the results of classification, which has led to significant results, especially when data can be labelled at the presence of training data. In this paper, firstly, altimeter and radiometric features are extracted from the LiDAR data and the point cloud derived from digital photogrammetry. Then, these features are participated in a classification process using SVM learning and random forest methods, and the ground and Non-ground points are classified. The classification results using this method on LiDAR data show a total error of $6.2 \%$, a type I error of $5.4 \%$, and a type II error of $13.2 \%$. The comparison of the proposed method with the results of LASTools software shows a reduction in total error and type I error (while increasing the type II error). This method was also investigated on the dense point cloud obtained from digital photogrammetry and based on this study, the total was $7.2 \%$, the type I error was $6.8 \%$, and the type II error was $10.9 \%$.
\end{abstract}

\section{INTRUDUCTION}

\subsection{Background}

Photogrammetry, with an age of more than a hundred years, has been used as one of the world's largest spatial data sources due to the advancements of high resolution digital areal camera, [1]. The preparation of 3D maps, Orthophoto, DTM Generation and High Resolution Point Cloud Extraction include the digital photogrammetry achievements, and with advancements in technology, its automation chain has been completed [2]. In addition to digital photogrammetric methods, processing and deploying LiDAR data has been widely studied with its extensive applications, including DTM extraction.

Three-dimensional point cloud derived from digital photogrammetry and LiDAR include ground and non-ground points, and therefore the first step in the production of digital ground model from point cloud data, includes the identification of ground points and the removal of non-ground points, which is called point cloud filtering, which its automation is faced with challenges due to the diversity of terrain features and topography [3-5]. Various methods have been suggested by different researchers to distinguish between ground and nonground points in the point cloud data. The disadvantage of most fully automated methods for identifying ground and non-ground points is for a particular type of ground. In other words, most of these algorithms have good outcomes in simple landscapes and encounter problems in complex landscapes. [6]. Obviously, region type important for the type of algorithm used, in terms of feature diversification and density of regions such as urban, non-urban, forest, sloped, flat, etc., and will affect the amount of automation of operations. In spite of extensive research on cloud computing, extraction of a digital ground model from large data with complex landscape is still a challenge for researchers [7, 8]. For example, the use of a limited set of parameters does not yield proper results for ground with discrete and complex surfaces, as well as different features.

\subsection{Related Work}

Various methods have been proposed by different researchers to distinguish non-ground and ground points in the point cloud data. When using these methods, considerations should be made such as the details of performed preprocessing, structure of input data, and other influential parameters [9]. The methods for filtering point cloud data according to performance are divided into several categories: rule-base methods, novel learning methods. [10, 11]

Rule base filtering methods include methods that are mainly based on the physical behavior of non-ground features. This group can be categorized to slope-based methods, methods based on mathematical morphology, baseline methods, (TIN) triangulated irregular network based methods, segmentation and clustering methods, and other methods which are mainly improvements of the above or independently have a special feature.

In recent years, machine learning algorithms have been widely used to classify images. In modern methods, geometric and radiometric features of each point can be used to trained classification.

\footnotetext{
* Corresponding author
} 
The results of learning methods performance in the filtering topic show that these methods are more effective than the Rule Based algorithms and represent the high potential of learningbased approaches in point cloud filtering. Extracting highly efficient and effective features for use in machine learning methods with the objective of classifying ground and nonground points seems to have better results. [12-14].

Chehata has used four features of anisotropy, planarity, sphereocity and Linearity for the implementation of random forest method. These features are derived based on the Eigen values of the variance-covariance matrix calculated for a neighborhood window with a given radius. In addition to these four features, altitude and recursive pulse characteristics are also used to train this method. The random forest classification method in this research has achieved a total accuracy of $94.35 \%$ which is a good result compared to Rule Based methods [15]. Niemeyer has used the Conditional Random Field (CRF) method to classify point cloud and made a complete study of classification using classifiers, features, and different neighborhoods. Most of the investigative methods in this research are monitored and not only classify clusters into ground and non-ground classes, but also classify many other classes. The CRF method provides the ability to combine textual information and learn specific communications of ground objects in the training phase. Therefore, this is a powerful algorithm for obtaining reliable results even in complex urban landscapes. [16].

The results of the performance of learning methods in the filtering topic show that these methods are more effective than the Rule Based algorithms and represent the high potential of learning-based approaches in point cloud filtering. The extraction of high-performance effective features for use in machine learning methods leads to better results in the classification of ground and non-ground points. [12-14]

\section{PROPOSED METHOD}

\subsection{Machine learning algorithm}

The machine learning techniques are a subset of artificial intelligence and, using these techniques, the computer learns the patterns available in the features and can use them. Nowadays, the use of machine learning techniques has been significantly developed in improving the classification results, which has led to significant results in the application of these methods, especially for data which can be labelled as training data.

In this paper, two SVM and random forest methods have been used for this purpose.

\subsubsection{SVM Algorithm}

The SVM algorithm has been developed as one of the solutions to machine learning. SVM performs its predictions using a linear combination of the kernel function which acts on a set of training data, namely the support vectors. The method presented by SVM is different than comparable methods such as neural networks; SVM training always finds the global minimum. The features of an SVM are greatly related to its kernel selection.

\subsubsection{Random Forest Algorithm}

Random forest algorithm is also an ensemble learning algorithm that uses decision trees for its simple and weak algorithms. In the random forest algorithm, several decision trees are used. In fact, a set of decision trees produce a forest together and this forest can make better decisions. To classify a data based on its characteristics, the data is given to each tree, and a tree is asked to "vote" for that class. The forest selects the classification that has the highest vote for all forest trees.

The decision trees were introduced in 2001 as one of the machine learning methods that combines the prediction of multiple single algorithms together using rule based methods. [18]

Random forest is a very useful and easy to use algorithm, because its default hyperparameters often produce good predictive results. Also, the number of its hyperparameters is not high and is easy to understand. [19]

One of the biggest problems in machine learning is overprocessing, but it often does not happen as easily as it happens for random forest categorizer. In general, training these algorithms is quickly achieved.

Another feature of the random forest is that the trees grow without pruning, and in this way, training does not affect the accuracy of the model too much and makes it simpler in terms of computation.

The points of the study area are classified using the extracted features and the random forest classification method into two classes of ground and non-ground points.

\subsection{Feature extraction}

Extraction is the first step in any learning system to create an input vector to the classification algorithm. To do this, first, the proper attributes should be extracted from the point cloud, to be used in the learning algorithm. The type of features has a direct impact on the classification result. In this research, two types of features are extracted from point cloud data: point features and neighborhood features. [20]

Point features are individually derived from information of each point, such as altitude, density, color information about the point cloud derived from optical sensors, and IR band values and other radiometric information in case of available multispectral and hyperspectral resources.

Neighborhood features include the topographic and radiometric information of the nearest neighboring points that indicate the morphology related to the shape of the ground or its features at that point. It also extracts statistical information about neighboring points such as minimum and maximum altitudes, as well as mean and standard deviation of points' altitude. According to the data studied in this research, eight features including five point features (two elevation features and three radiometric features) and three neighborhood features related to points altitude are extracted from the point cloud data. [21, 22] Table 1 shows the extracted features from the point cloud in this research.

Regarding the investigation of the implementation of the proposed algorithm on two types of LiDAR data and the point cloud derived from a digital photogrammetric, in this paper and in the LiDAR data, only geometric features and in the point cloud, all eight features are extracted.

\subsection{Training \& Testing}

After extracting the feature, the sample data from each of the ground and non-ground classes are randomly selected. Here, $40 \%$ of the data is considered as training data for designing the classifier of machine learning and $60 \%$ of the data as check data for investigating the classifier accuracy. Choosing the right training data is very important in achieving the proper accuracy. 


\begin{tabular}{|c|c|c|c|}
\hline \multicolumn{2}{|c|}{ Feature Type } & Feature Name & Function \\
\hline \multirow{5}{*}{ Point Features } & \multirow{2}{*}{ Geometry } & Normalized Height 1 & normalized height_1 $=\frac{\left(h-h_{\min }^{L}\right)}{\left(h_{\max }^{L}-h_{\min }^{L}\right)}$ \\
\hline & & Normalized Height 2 & normalized height_2 $=\frac{\left(h-\bar{h}^{G}\right)}{\sigma_{h}^{G}}$ \\
\hline & \multirow{3}{*}{ Radiometric } & Normalized Intensity 1 & normalized intensity_1 $=\frac{\sigma_{i}^{L}}{\bar{i}^{L}}$ \\
\hline & & Normalized Intensity 2 & normalized intensity_2 $=\frac{\left(i-\bar{i}^{G}\right)}{\sigma_{i}^{G}}$ \\
\hline & & NDVI & $\mathrm{NDVI}=\frac{\rho_{\mathrm{nir}}-\rho_{\mathrm{red}}}{\rho_{\mathrm{nir}}+\rho_{\mathrm{red}}}$ \\
\hline \multirow{3}{*}{$\begin{array}{l}\text { Neighborhood } \\
\text { Features }\end{array}$} & \multirow{3}{*}{ Geometry } & Slop & Slope $=\frac{\Delta Z}{\Delta X}$ Slope $=\frac{\Delta Z}{\Delta X}$ \\
\hline & & Roughness & $\mathrm{Ra}=1 / N . \sum_{n=1} \mid h_{n}$ \\
\hline & & Curvature & $c=x(s)+t$ \\
\hline
\end{tabular}

Table 1. the extracted features from point cloud

\section{DATASET}

In this paper, the results of the proposed method are examined on two datasets: ISPRS Filter Test Dataset and Dehbar Dataset ISPRS Filter Test dataset is a LIDAR data and Dehbar dataset is a point cloud derived from digital photogrammetry.

\subsection{ISPRS Filter Test dataset}

The ISPRS dataset contains 15 LiDAR data sample areas. The ISPRS dataset has 15 sample areas. From the 15 samples, ten sample areas were chosen for training and five sample areas for testing. Figure 6 shows the five testing samples.

The dataset has a low point density. This causes the terrain to be poorly represented, especially on a combination of steep terrain and low vegetation. There are only two returns, first and last, in contrast to five multiple returns on a typical modern LIDAR point cloud. Fewer returns mean less information is available to separate vegetation from the ground. This situation makes the dataset more challenging for ground classification.

\subsection{Dehbar Dataset}

The Dehbar dataset refers to mountainous areas with forest and non-forest areas as well as residential areas. This data is based on digital $10-\mathrm{cm}$ res. aerial photographs, which, after photogrammetric steps, are prepared through digital photogrammetric processing and photo synchronization operation on covered pair images, and finally a colored point cloud is extracted. In addition to elevation data, Orthophoto has been prepared in the 4-band IRGB and used to extract radiometric features. Collection of information on the classification of the dataset points is carried out manually by the expert agent, so that all the points of the point cloud are labelled and it is recognized that they are whether related to bare earth or are non-ground. Figure 2 shows a part of the Dehbar dataset. a. Sample 11

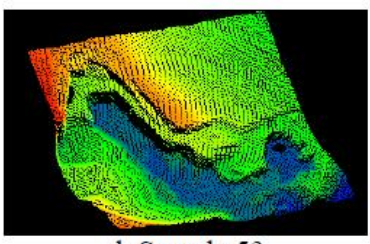

d. Sample 53

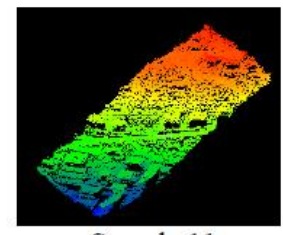

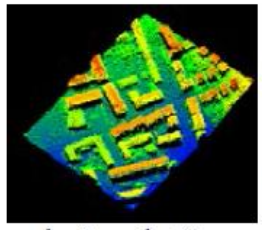

b. Sample 12

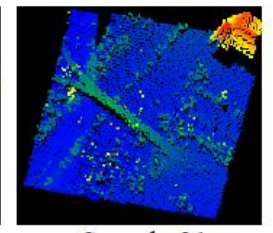

c. Sample 21

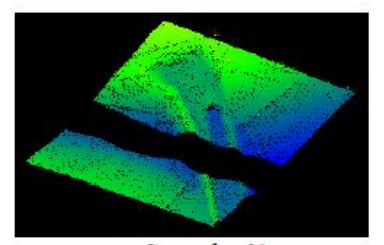

e. Sample 61
Figure 1. ISPRS testing samples: (a) steep terrain, (b) dense buildings, (c) bridge, (d) height discontinuity and (e) embankment and data gap

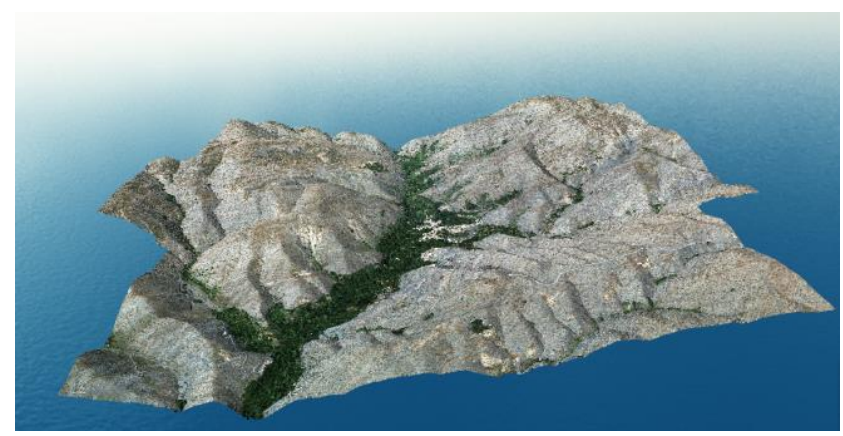

Figure 2. Dehbar dataset 


\section{RESULT AND DESCUSION}

This section explains the results of the implementation of SVM and RF algorithms, the evaluation of accuracy and comparison of computational costs. The results are compared with the classification derived from the LAStools software, which uses the TIN method for filtering and is considered to be the best filtering method.

In this assessment, the total error (false points of class), type I error (rejection of ground points), type II error (acceptation of non-ground points as ground points) were investigated.

The results of implementing the proposed method on the ISPRS dataset show an improvement in the total error and type I error, but has a higher type II error. Therefore, the implementation of this method can significantly categorize non-ground and ground points at a higher rate and low cost. Comparison of the results of this method with the rule-based methods is shown in Table 2. Table 3 also shows the results of performing SVM and RF algorithms on the Dehbar dataset derived from digital photogrammetry compared to the rule based method.

The results of classification on LiDAR data using this method show a total error of $6.2 \%$, a type I error of $5.4 \%$, and a type II error of $13.2 \%$. The comparison of the proposed method with the results of LASTools software shows a reduction in total error and type I error (while increasing the type II error).

Sample 11 is the most difficult region to deal with, in contrast to other samples: Steep lands with low vegetation and buildings. The RF algorithm method can properly eliminate buildings and vegetation in the upper and middle zone without losing ground points. However, some buildings can not be properly removed in the downstream area.

Sample 12 includes flat land with dense buildings. In general, RF can be performed in this type of area. Sample 21 has a bridge and a large building. The proposed method works well, especially as the bridge and large building are removed.

Sample 53 has a disturbance in altitude, while sample 61 has a gap region. The ground points in both samples can be missclassified as non-ground points due to their shape. In sample 53, almost all break lines are taken correctly with the proposed method. This type of land is often identified with filters that locally remove non-ground points. However, RF can not classify a few non-ground points in samples 53 and 61. Due to the limited number of non-ground points compared to ground points, the second line is relatively large.

\begin{tabular}{|c|c|c|c|}
\hline \multirow{2}{*}{ Sample } & \multicolumn{3}{|c|}{ Total Error } \\
\cline { 2 - 4 } & RF & SVM & LASTools \\
\hline Samp11 & 15.7 & 16.8 & 17.7 \\
\hline Samp12 & 4.2 & 8.3 & 7 \\
\hline Samp21 & 2.7 & 3.4 & 6.7 \\
\hline Samp53 & 5.5 & 6.5 & 14.4 \\
\hline Samp61 & 2.9 & 5.1 & 17.2 \\
\hline Average & 6.2 & 8.0 & 12.6 \\
\hline
\end{tabular}

\begin{tabular}{|c|c|c|c|}
\hline \multirow{2}{*}{ Sample } & \multicolumn{3}{|c|}{ Type I Error } \\
\cline { 2 - 4 } & RF & SVM & LASTools \\
\hline Samp11 & 14.6 & 27.5 & 26.9 \\
\hline Samp12 & 4.2 & 13.2 & 12.9 \\
\hline Samp21 & 1.4 & 3.1 & 8 \\
\hline Samp53 & 4.9 & 5.6 & 14.8 \\
\hline Samp61 & 1.7 & 4.9 & 17.8 \\
\hline Average & 5.4 & 10.9 & 16.1 \\
\hline
\end{tabular}

\begin{tabular}{|c|c|c|c|}
\hline \multirow{2}{*}{ Sample } & \multicolumn{3}{|c|}{ Type II Error } \\
\cline { 2 - 4 } & RF & SVM & LASTools \\
\hline Samp11 & 16.1 & 10.2 & 5.2 \\
\hline Samp12 & 5.6 & 2.4 & 0.8 \\
\hline Samp21 & 7.4 & 5.6 & 1.9 \\
\hline Samp53 & 20.3 & 27.5 & 3.2 \\
\hline Samp61 & 16.4 & 10.3 & 0.4 \\
\hline Average & 13.2 & 11.2 & 2.3 \\
\hline
\end{tabular}

Table 2. A comparison between the accuracies obtained by the proposed method and LAStools on ISPRS dataset.

This method was also investigated on the dense point cloud obtained from digital photogrammetry and based on this study, the total error was $7.2 \%$, type I error was $6.8 \%$, and type II error was $10.9 \%$ that shown in Table 3 .

In general, the proposed method works well to minimize total error and type I error, but type II error is higher than other methods.

\begin{tabular}{|c|c|c|c|}
\hline \multirow{2}{*}{ Dehbar Sample } & \multicolumn{3}{|c|}{ Total Error } \\
\cline { 2 - 4 } & RF & SVM & LASTools \\
\hline Total Error & 7.2 & 8.1 & 13.4 \\
\hline Type I Error & 6.8 & 7.3 & 11.6 \\
\hline Type II Error & 10.9 & 12.3 & 6.2 \\
\hline
\end{tabular}

Table 3: A comparison between the accuracies obtained by the proposed method and LAStools on Dehbar dataset.

\section{CONCLUSION AND FERTHER WORK}

Over the past few decades, point cloud filtering techniques have been widely studied and various algorithms have been proposed with a different approach for different ground conditions. However, given the variety of filtering techniques available, the simple use of a filtering method can hardly be useful for different terrains. In this paper, while reviewing traditional methods and algorithms, recent advances in point cloud filtering, especially the use of new learning methods, have been studied. The filtering methods in this paper are divided into two main groups of traditional methods, which are mainly rule based, and are designed based on the physical behavior of ground and non-ground points, and novel methods, which mainly identify land and non-terrestrial points, based on machine learning algorithms.

The main advantages of traditional methods include the theoretical support and the regularity of the methods, the use of specific methods for particular regions, and the presentation of appropriate results by some methods for a main part of input data and the challenges of these methods include the suitability of each method for certain types of land, dependence on the adjustment of various parameters.

Advantages of novel methods include the possibility of simultaneous application in different types of land, need for parameter adjustment, fast performance for high volume data, development and evolution of learning methods for higher accuracy and correctness. The lack of theoretical support, need for high training data, dependence of the results on training data, are among the weaknesses of the novel methods. 
In this paper, we compare the performance of machine learning methods with rule-based methods and better performance of these methods is revealed in comparison with traditional methods. Due to the importance of the features used in learning algorithms, the use of deep learning methods is recommended for automatic extraction of the feature.

In the end, the main strategy of this paper, which includes the previous three strategies, is the use of novel deep learning methods, in which, besides the possibility of using various information resources, it is possible to apply preprocessing methods. For example, performing some traditional methods on data and focusing on data and points with complexities, and then using machine learning methods to speed up is suggested.

\section{REFERENCES}

[1] K. Nurminen et al., "Automation Aspects for the Georeferencing of Photogrammetric Aerial Image Archives in Forested Scenes," Remote Sensing, vol. 7, no. 2, pp. 15651593, 2015/02/02 2015.

[2] C. Heipke, M. Madden, Z. Li, and I. Dowman, "Theme issue "State-of-the-art in photogrammetry, remote sensing and spatial information science"," ISPRS Journal of Photogrammetry and Remote Sensing, vol. 115, pp. 1-2, 2016/05 2016.

[3] G. Sithole and G. Vosselman, "Experimental comparison of filter algorithms for bare-Earth extraction from airborne laser scanning point clouds," ISPRS Journal of Photogrammetry and Remote Sensing, vol. 59, no. 1-2, pp. 85-101, 2004/08 2004.

[4] K. Zhang and D. Whitman, "Comparison of Three Algorithms for Filtering Airborne Lidar Data," Photogrammetric Engineering \& Remote Sensing, vol. 71, no. 3, pp. 313-324, 2005/03/01 2005.

[5] M. Bartels and H. Wei, "Threshold-free object and ground point separation in LIDAR data," Pattern Recognition Letters, vol. 31, no. 10, pp. 1089-1099, 2010/07 2010.

[6] X. Liu, "Airborne LiDAR for DEM generation: some critical issues," Progress in Physical Geography, vol. 32, no. 1, pp. 31-49, 2008/02/01 2008.

[7] Z. Chen, B. Devereux, B. Gao, and G. Amable, "Upwardfusion urban DTM generating method using airborne Lidar data," ISPRS journal of photogrammetry and remote sensing, vol. 72, pp. 121-130, 2012.

[8] L. Wei-Lwun, K. P. Murphy, J. J. Little, A. Sheffer, and F. Hongbo, "A Hybrid Conditional Random Field for Estimating the Underlying Ground Surface From Airborne LiDAR Data," IEEE Trans. Geosci. Remote Sensing, vol. 47, no. 8, pp. 29132922, 2009/08 2009

[9] X. Meng, L. Wang, J. L. Silván-Cárdenas, and N. Currit, "A multi-directional ground filtering algorithm for airborne LIDAR," ISPRS Journal of Photogrammetry and Remote Sensing, vol. 64, no. 1, pp. 117-124, 2009.

[10] G. Sithole and G. Vosselman, "Comparison of filtering algorithms," in Proceedings of the ISPRS working group III/3 workshop, 2003, pp. 71-78.

[11] X. Meng, N. Currit, and K. Zhao, "Ground Filtering Algorithms for Airborne LiDAR Data: A Review of Critical Issues," Remote Sensing, vol. 2, no. 3, pp. 833-860, 2010.

[12] A. Rizaldy, C. Persello, C. Gevaert, and S. Oude Elberink, "FULLY CONVOLUTIONAL NETWORKS FOR GROUND CLASSIFICATION FROM LIDAR POINT CLOUDS," ISPRS Annals of Photogrammetry, Remote Sensing \& Spatial Information Sciences, vol. 4, no. 2, 2018.

[13] C. Gevaert, C. Persello, F. Nex, and G. Vosselman, "A deep learning approach to DTM extraction from imagery using rule-based training labels," ISPRS Journal of
Photogrammetry and Remote Sensing, vol. 142, pp. 106-123, 2018.

[14] X. Hu and Y. Yuan, "Deep-learning-based classification for DTM extraction from ALS point cloud," Remote sensing, vol. 8, no. 9, p. 730, 2016.

[15] N. Chehata, L. Guo, and C. Mallet, "Airborne lidar feature selection for urban classification using random forests," International Archives of Photogrammetry, Remote Sensing and Spatial Information Sciences, vol. 38, no. Part 3, p. W8, 2009.

[16] J. Niemeyer, F. Rottensteiner, and U. Soergel, "Conditional random fields for lidar point cloud classification in complex urban areas," ISPRS annals of the photogrammetry, remote sensing and spatial information sciences, vol. 3, pp. 263$268,2012$.

[17] J. Zhang, X. Lin, and X. Ning, "SVM-based classification of segmented airborne LiDAR point clouds in urban areas," Remote Sensing, vol. 5, no. 8, pp. 3749-3775, 2013.

[18] L. Breiman, "Random forests," Machine learning, vol. 45 , no. 1 , pp. 5-32, 2001.

[19] S. Kotsiantis and P. Pintelas, "Combining bagging and boosting," International Journal of Computational Intelligence, vol. 1, no. 4, pp. 324-333, 2004.

[20] S. H. H. Nourzad and A. Pradhan, "Binary and multiclass classification of fused LIDAR-imagery data using an ensemble method," in Construction Research Congress, 2012, pp. 909-918.

[21] S. H. H. Nourzad and A. Pradhan, "Ensemble methods for binary classifications of airborne LiDAR data," Journal of Computing in Civil Engineering, vol. 28, no. 6, p. 04014021, 2012.

[22] S. K. Lodha, D. M. Fitzpatrick, and D. P. Helmbold, "Aerial lidar data classification using adaboost," in 3-D Digital Imaging and Modeling, 2007. 3DIM'07. Sixth International Conference on, 2007: IEEE, pp. 435-442. 